\title{
Derivación biliodigestiva guiada por ultrasonografía endoscópica en el paciente con adenocarcinoma de páncreas y obstrucción de la vía biliar estadio IV: reporte de caso
}

\section{Biliodigestive shunt guided by endoscopic ultrasonography in patient with stage IV pancreatic adenocarcinoma and obstruction of the bile duct. A case report}

\author{
María Manrique, ${ }^{1}$ 이 Wilmar Martín-M.2*
}

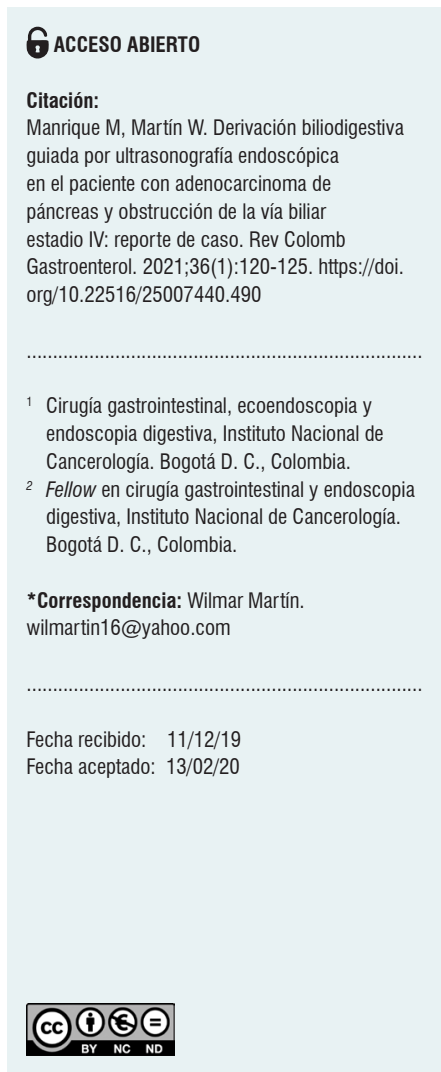

\section{Resumen}

La endosonografía endoscópica es una alternativa que sirve como guía para la realización de derivaciones biliodigestivas en los casos en los que la colangiopancreatografía retrógrada endoscópica (CPRE) ha fallado. Se han descrito técnicas como la coledocoduodenostomía o coledocoantrostomía guiadas por ultrasonografía endoscópica (USE).

Se describe el caso de una paciente de 72 años con adenocarcinoma de páncreas, compromiso portal y de paredes duodenales, en quien se usó la CPRE para intentar una derivación paliativa y fue fallida, por lo cual se realizó la colocación de un stent metálico guiado por endosonografía endoscópica, con adecuada respuesta clínica al tratamiento. En conclusión, el procedimiento es seguro y la endosonografía es una vía alterna efectiva en los casos de CPRE fallidas para lograr derivaciones biliares en casos de obstrucción de la vía biliar de origen maligno.

\section{Palabras clave}

Obstrucción del conducto biliar, colangiopancreatografía endoscópica retrógrada (CPRE), ultrasonografía endoscópica (USE).

\begin{abstract}
Endoscopic endosonography is an alternative to guide biliodigestive shunting in cases where endoscopic retrograde cholangiopancreatography (ERCP) has failed. Techniques such as choledoduodenostomy or choledochoantrostomy guided by endoscopic ultrasonography have been described.

This is the case of a 72-year-old patient with pancreatic adenocarcinoma, portal vein and duodenal wall involvement, in whom ERCP to try a palliative shunt failed. Therefore, a metal stent was placed using endoscopic endosonography, with adequate clinical response to the procedure and treatment. It is concluded that the procedure is safe and that endosonography is an effective alternative in cases of ERCP failure to achieve biliary bypass in cases of malignant biliary obstruction.
\end{abstract}

Keywords

Bile duct obstruction, Retrograde endoscopic cholangiopancreatography (ERCP), Endoscopic ultrasonography (EUS).

\section{INTRODUCCIÓN}

La derivación biliar endoscópica es la técnica utilizada en pacientes con estenosis del confluente biliopancreático, secundaria a neoplasias irresecables, y la CPRE es la técnica de elección para llevar a cabo estas derivaciones (1). Aun siendo el método de elección, la CPRE falla entre un $5 \%$ y $10 \%$ de los casos (2), debido a situaciones tales como alte- 
ración de la anatomía, obstrucción gastroduodenal, segmento obstruido con longitud considerable, distorsión de la papila, divertículo periampular y stents duodenales (2). Históricamente, si la CPRE falla, el paciente es llevado a derivaciones percutáneas transparietohepáticas, y de igual forma se han observado con este procedimiento tasas de complicaciones y morbilidad que pueden llegar al $33 \%$ (2), entre las que se incluyen disfunción del catéter y colangitis asociada, fuga de bilis o sangrado a la cavidad peritoneal; y finalmente, la opción quirúrgica es la medida en caso de que las dos anteriores técnicas no logren derivar adecuadamente el conducto biliar, con la consecuente morbilidad de cualquier intervención quirúrgica. Por estas razones, la derivación biliar guiada por ultrasonografía endoscópica (USE) ha surgido como una alternativa de drenaje.

Se presenta el caso clínico de una paciente adulta mayor con obstrucción biliar de origen maligno, a quien se le practicó la inserción de un stent metálico guiado por USE después de una CPRE fallida para derivación.

\section{DESCRIPCIÓN DEL CASO}

Se trata de una paciente de 72 años con cuadro clínico de obstrucción biliar secundaria a una masa en la cabeza del páncreas, cuyos estudios se describen a continuación: los niveles de bilirrubinas iniciales totales se encontraron en $12,32 \mathrm{mg} / \mathrm{dL}$ a expensas de la directa, que es de $10,8 \mathrm{mg} /$ $\mathrm{dL}$; tomografía de abdomen total que reportó neoplasia en la cabeza del páncreas, rodeando en $180^{\circ}$ la vena mesenté-

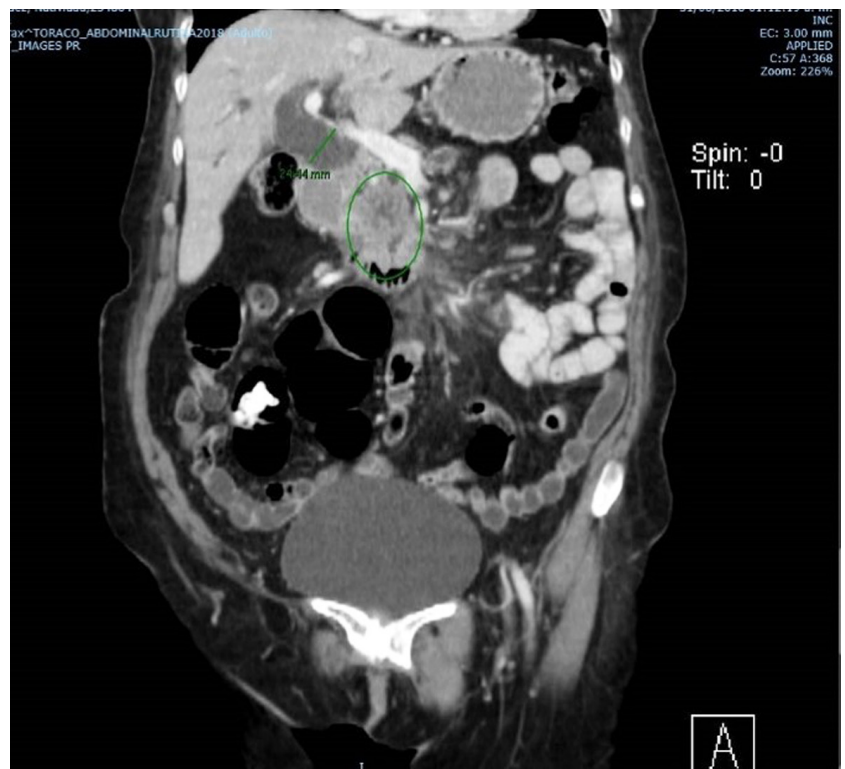

Figura 1. Masa de la cabeza del páncreas, obstrucción de la vía biliar distal. rica superior; confluente esplenoportal permeable; arteria hepática permeable; dilatación de la vía biliar intra- y extrahepática, y del conducto pancreático principal; aumento de la densidad de la grasa de la raíz del mesenterio con ganglios con ejes cortos (entre $5 \mathrm{~mm}$ y $6 \mathrm{~mm}$ ) sospechosos por su realce; y lesión focal hepática en el segmento 6 de aspecto sospechoso para infiltración neoplásica, sin liquido libre (Figuras 1 y 2 ).

Se complementaron estudios con resonancia de abdomen simple que mostró una dilatación de la vía biliar intray extrahepática asociada con una masa pancreática localizada en la cabeza, con signos de compromiso de la raíz del mesenterio y lesión focal hepática en el sexto segmento de aspecto sospechoso (Figura 3 ).

Con los hallazgos descritos se decidió el manejo paliativo y se llevó a CPRE con la intención de realizar una derivación biliar con stent; se encontró la papila distorsionada con retracción local de las paredes duodenales y se logró la canulación de la vía biliar distal, pero fue fallida para la vía biliar proximal. Entonces, se realizó la derivación biliodigestiva guiada por USE, en la que se encontró una infiltración neoplásica de la papila duodenal que impide su canulación y la cabeza del páncreas tenía una lesión hipoecoica heterogénea de $23 \mathrm{~mm}$ de diámetro con una dilatación retrógrada de la vía biliar hasta $18 \mathrm{~mm}$ (Figura 4). Se realizó una punción inicial de la masa para el estudio histológico y, posteriormente, de la vía biliar transduodenal con una aguja de $19 \mathrm{Fr}$, avanzando la guía hidrofílica 0,035; la dilatación se realizó con un balón dilatador biliar bajo

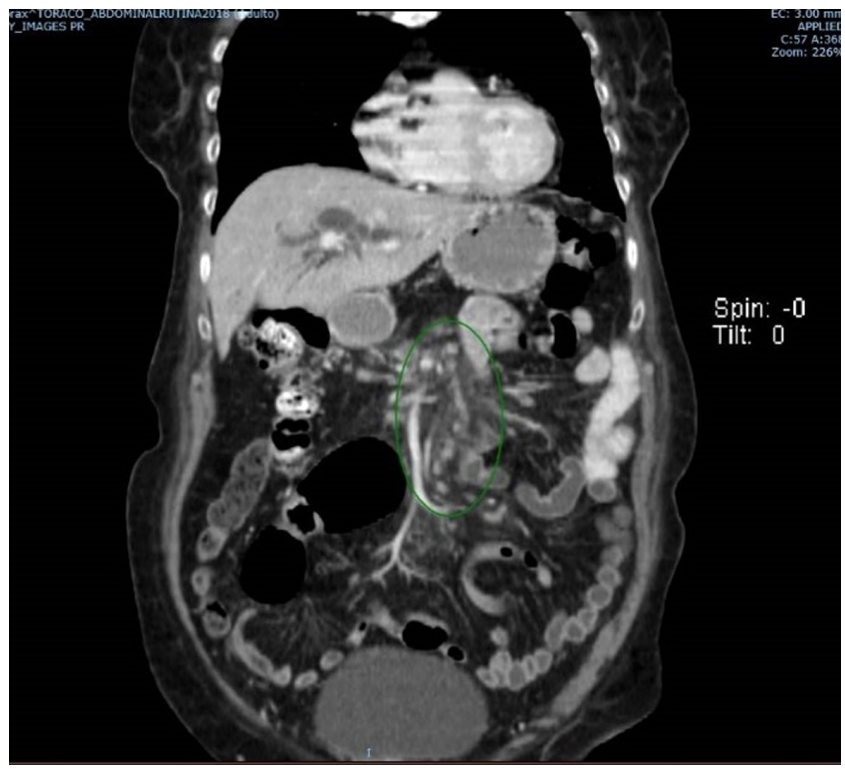

Figura 2. Aumento de la densidad de la grasa mesentérica con adenopatías subyacentes. 


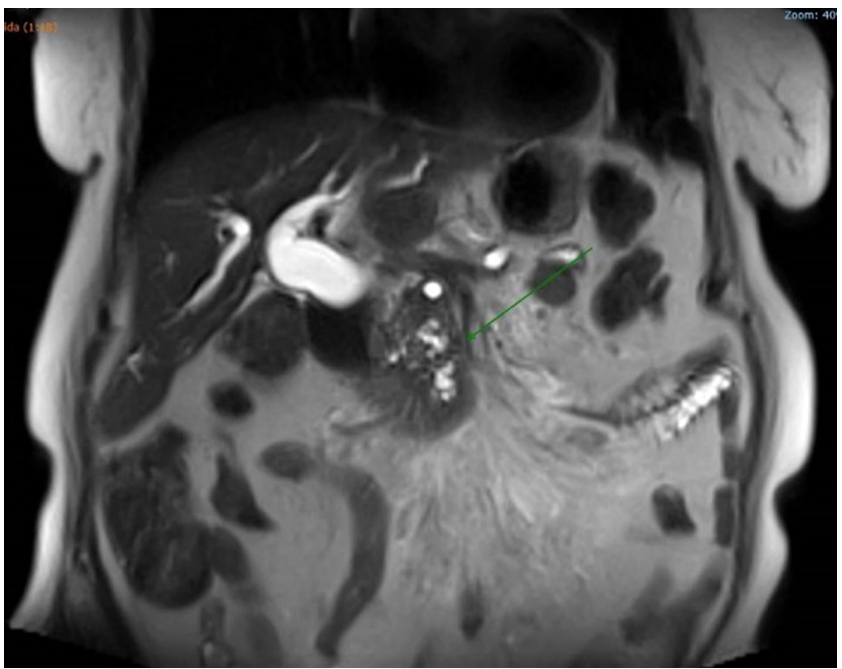

Figura 3. Masa de la cabeza del páncreas, dilatación de la vía biliar intray extrahepática.

control fluoroscópico y la posterior colocación del stent biliar metálico completamente cubierto de 10 x $80 \mathrm{~mm}$, el cual se liberó bajo control endoscópico y fluoroscópico con una adecuada apertura y drenaje del medio de contraste y de bilis clara, sin complicaciones posteriores (Figuras 5 y 6 ). El reporte de la citología obtenida por endosonografía

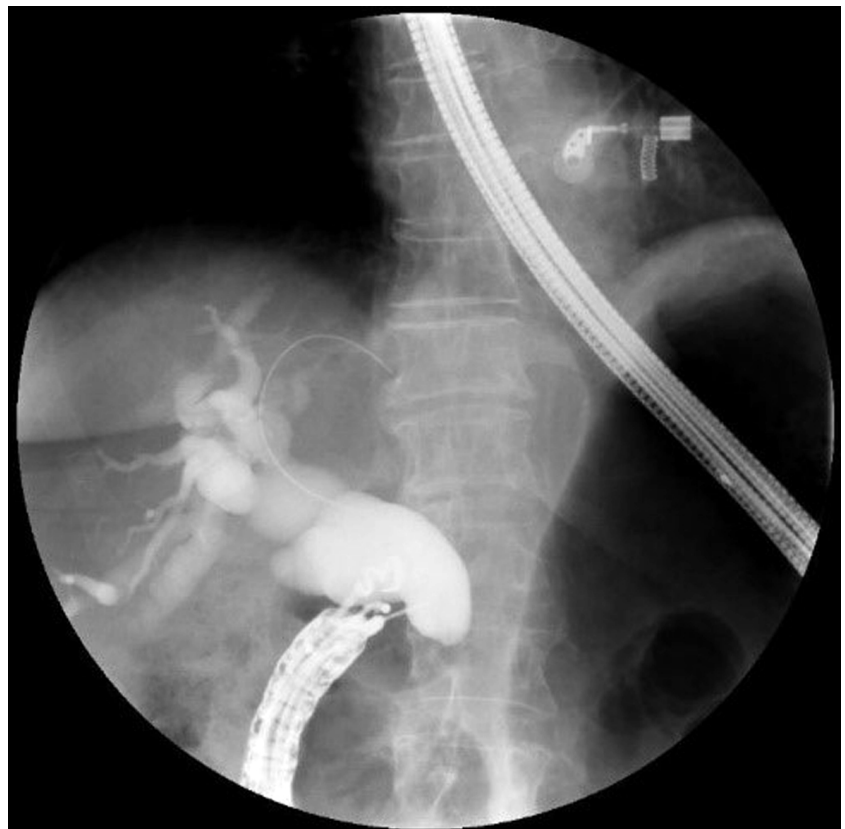

Figura 5. Canulación de la vía biliar guiada por endosonografía y fluoroscopia en la que se observa la dilatación con la amputación distal.

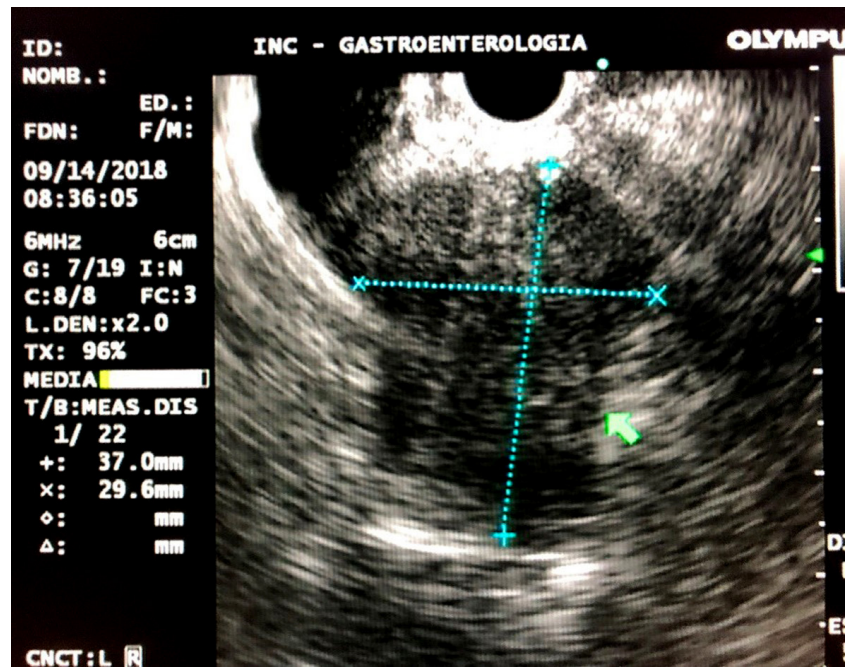

Figura 4. Visión por endosonografía de la masa en la cabeza de páncreas y dilatación de la vía biliar.

confirmó el compromiso por adenocarcinoma. A los 2 meses se le realizó control de bilirrubina total en $2,42 \mathrm{mg} /$ $\mathrm{dL}$; directa, en $1,85 \mathrm{mg} / \mathrm{dL}$; e indirecta, en $0,58 \mathrm{mg} / \mathrm{dL}$ (Figura 7). La paciente continuó el manejo con oncología y cuidados paliativos.

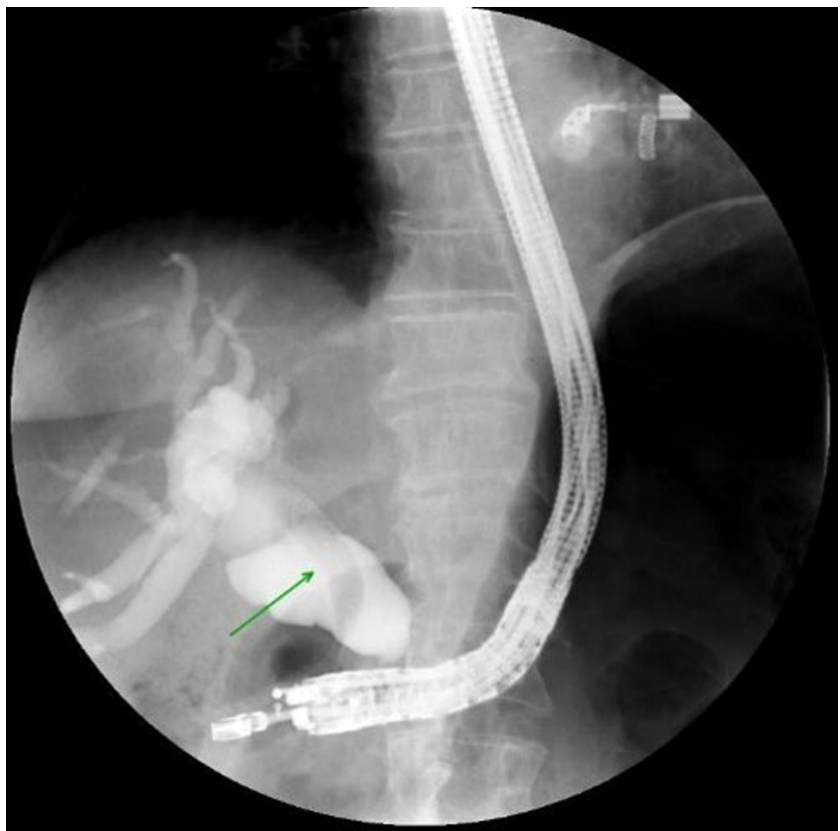

Figura 6. Stent metálico liberado bajo control por endosonografía y guía fluoroscópica. 


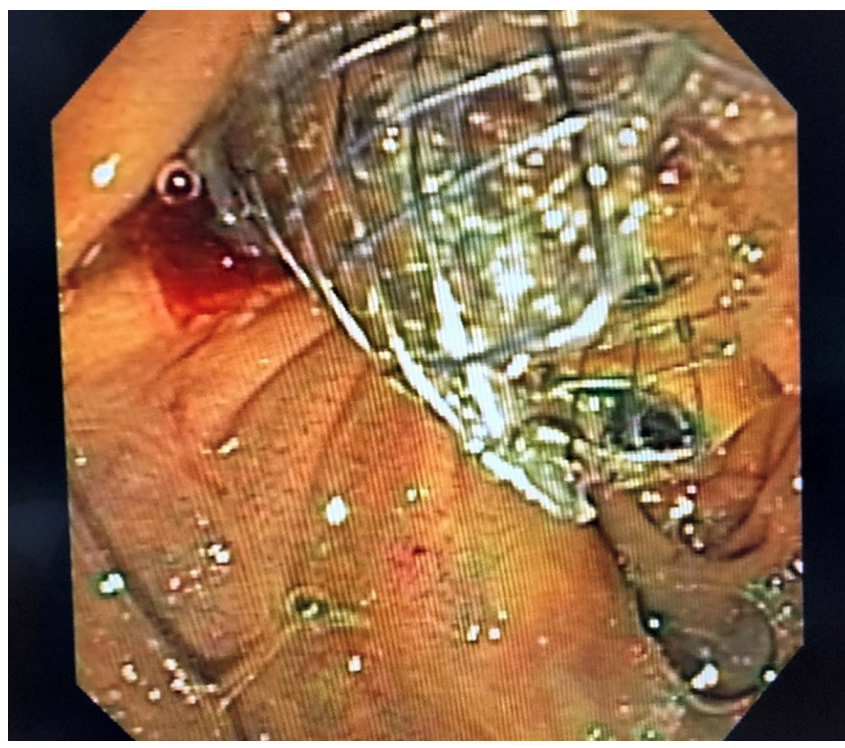

Figura 7. Resultado final de la derivación biliodigestiva guiada por USE.

\section{DISCUSIÓN}

El caso corresponde a obstrucción del colédoco distal de origen maligno secundario a adenocarcinoma inoperable en la cabeza del páncreas, que requirió de derivación de la vía biliar dados los efectos de la compresión. $\mathrm{Al}$ respecto se han documentado casos semejantes en los que por el carácter compresivo de patologías malignas no es posible canular la vía biliar, pero lo mismo también se presenta en obstrucciones de origen benigno (3-6).

La derivación biliodigestiva guiada por USE es una alternativa cuando la CPRE falla en el acceso a la papila o la vía biliar, lo cual sucede entre un $5 \%$ y un $10 \%$ (7); en fallas ocasionadas por alteración anatómica, como en el presente caso; o frente a distorsión ampular, diverticulosis periampular, obstrucción de la salida gástrica o stents duodenales in situ (2).

La derivación biliar transparietohepática se realiza cuando la CPRE falla, pero presenta hasta un $33 \%$ de eventos adversos: hemorragias, bilioperitoneo, infección y colangitis aguda, entre otros (8); mientras que la derivación biliodigestiva guiada por USE representa un menor riesgo de este tipo de eventos.

Los drenajes transparietohepáticos están asociados con una morbilidad importante. Hay reportes de complicaciones que llegan hasta el $60 \%$ en las derivaciones transparietohepáticas, comparadas con el $35 \%$ de las realizadas por derivación biliodigestiva guiada por USE (3).
La derivación biliodigestiva guiada por USE fue mencionada inicialmente por Giovannini y colaboradores en 2001 (9), y actualmente se está demostrando su seguridad y eficacia dado el abordaje de esta técnica (2), adicional a las ventajas que presenta al ser mínimamente invasiva, poderse realizar posteriormente a una CPRE fallida en el mismo tiempo del procedimiento y por el mismo especialista, lograr el drenaje de los conductos biliares intra- $y$ extrahepáticos, producir dolor leve por el procedimiento o sin la presencia de este, no implicar drenaje externo, dislocar el drenaje, no limitar las actividades diarias del paciente, requerir solo un tiempo leve de hospitalización y presentar una baja tasa de complicaciones $(3,10,11)$.

Con respecto a las complicaciones, se menciona que varían entre un $3,4 \%$ y un $38,6 \%$, con una tasa promedio entre $17 \%$ y $18,9 \%(3,12,13)$, pero con tendencia a ser menores con el tiempo $(10,13-15)$, y comprenden fuga biliar (3\%), hemorragia $(2,7 \%)$, colangitis $(0,3 \%)$, sepsis y peritonitis $(3,5 \%)(10)$. Dentro de las complicaciones graves, se han descrito con una muy baja frecuencia la migración del stent en la cavidad peritoneal y perforaciones fatales $(10,16,17)$; sin embargo, no se ha definido aún un consenso al respecto de las complicaciones (2).

Como se mencionó, las derivaciones percutáneas están asociadas con un menor éxito clínico y, por consiguiente, un mayor costo. Con respecto a la comparación entre CPRE frente a la USE para derivación biliar, ambos procedimientos tienen la misma efectividad, pero no hay estudios prospectivos que comparen la derivación biliodigestiva guiada por USE como procedimiento primario en la derivación biliodigestiva (2).

En los casos de obstrucción maligna, puede ser bastante compleja la canulación de la papila y, por la misma razón, se incrementa el tiempo de manipulación, lo cual puede desencadenar eventos adversos, de los cuales el principal es la pancreatitis posterior a la CPRE, que puede llegar hasta el $10 \%$ en los casos que el tiempo de canulación excede los 10 minutos (18).

En este sentido, en el último consenso multinstitucional se definió que la derivación biliodigestiva guiada por USE es la técnica de elección para pacientes con una obstrucción maligna de la vía biliar y que no fue posible derivarlos con la realización de CPRE, para lo cual han propuesto un algoritmo de manejo (Figura 8) (1).

Se ha demostrado que es un procedimiento seguro y efectivo para el manejo de la obstrucción maligna, con resultados muy similares al compararlo con la CPRE. Inclusive, se puede pensar que no solo es la elección en casos de una CPRE fallida, lo puede ser también en casos de obstrucción 


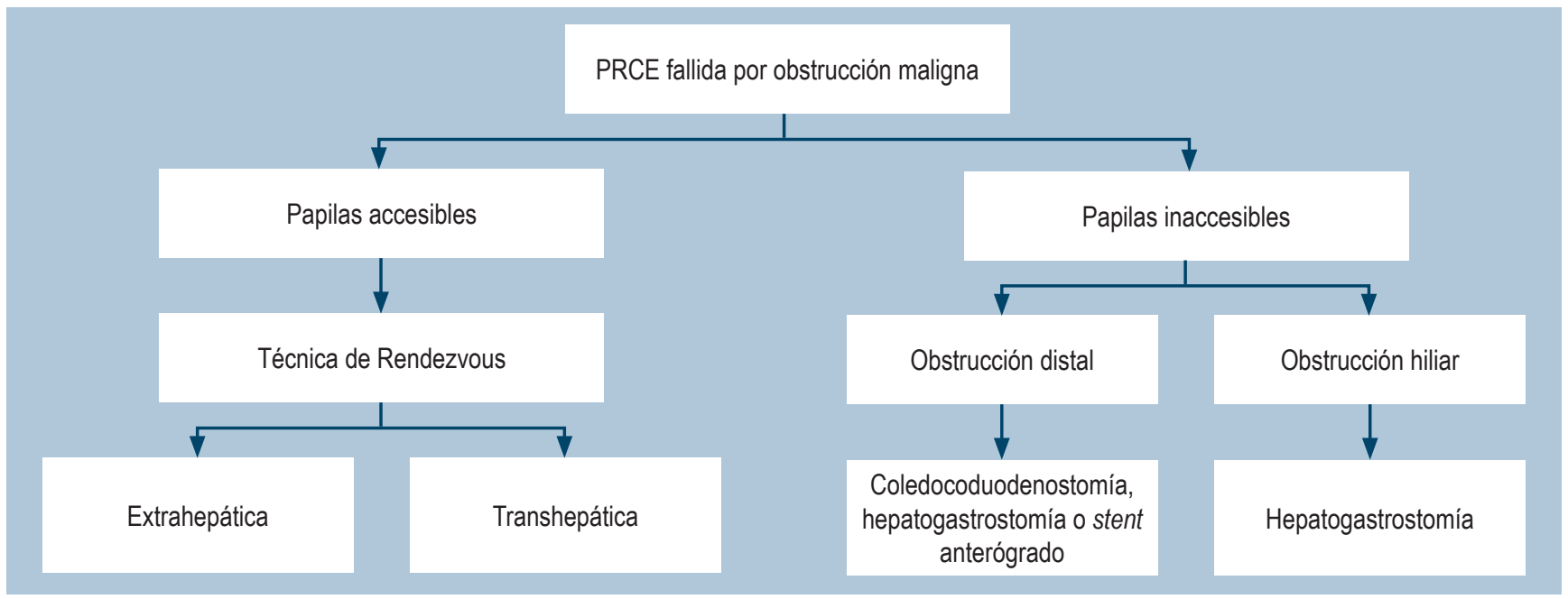

Figura 8. Algoritmo de manejo para los pacientes con CPRE fallida por una obstrucción maligna (1). Tomado de: Guo J et al. Endosc Ultrasound. 2018;7(6):356-365.

gástrica distal; y gracias a que se realiza bajo visión directa, se puede evitar el riesgo de pancreatitis aguda.

\section{CONCLUSIÓN}

La derivación biliodigestiva guiada por endosonografía endoscópica es una técnica emergente, considerada una muy buena alternativa en aquellos casos en los que la CPRE como procedimiento inicial resultó fallido. El procedimiento es práctico, efectivo, eficaz y eficiente, con un adecuado margen de seguridad y respuesta clínica. Al ser una técnica emergente, se requieren de mayores estudios que permitan consolidar las ventajas referidas.

\section{Fuentes de financiación}

Los autores expresan que el presente estudio fue financiado con recursos propios y no implicó aportes externos de ninguna índole.

\section{Conflictos de interés}

Los autores expresan no presentar ningún tipo de conflicto de interés con el presente estudio.

\section{REFERENCIAS}

1. Guo J, Giovannini M, Sahai AV, Saftoiu A, Dietrich CF, Santo E, Fusaroli P, Siddiqui AA, Bhutani MS, Bun Teoh AY, Irisawa A, Arturo Arias BL, Achanta CR, Jenssen C, Seo DW, Adler DG, Kalaitzakis E, Artifon E, Itokawa F, Poley JW, Mishra G, Ho KY, Wang HP, Okasha HH, Lachter J, Vila JJ, Iglesias-Garcia J, Yamao K, Yasuda K, Kubota K, Palazzo L, Sabbagh LC, Sharma M, Kida M, El-Nady M, Nguyen NQ, Vilmann P, Garg PK, Rai P, Mukai S, Carrara S, Parupudi S, Sridhar S, Lakhtakia S, Rana SS, Ogura T, Baron TH, Dhir V, Sun S. A multi-institution consensus on how to perform EUS-guided biliary drainage for malignant biliary obstruction. Endosc Ultrasound. 2018;7(6):356365. https://doi.org/10.4103/eus.eus_53_18

2. Baars JE, Kaffes AJ, Saxena P. EUS-guided biliary drainage: A comprehensive review of the literature. Endosc Ultrasound. 2018;7(1):4-9. https://doi.org/10.4103/eus. eus $105 \quad 17$

3. Giovannini M, Bories E, Napoleon B, Barthet M, Caillol F, Pesenti C. Multicenter randomized phase II study: Percutaneous biliary drainage vs. EUS guided biliary drainage: Results of intermediate analysis. Gastrointest Endosc 2015;81(5 Suppl):AB174. https://doi.org/10.1016/j. gie.2015.03.1926 
4. Du L, D’Souza P, Thiesen A, Girgis S, Owen R, McNally D, Sarlieve P, Sandha G. Percutaneous transhepatic cholangioscopy for indeterminate biliary strictures using the SpyGlass system: a case series. Endoscopy. 2015;47(11):1054-6. https://doi. org/10.1055/s-0034-1392527

5. Franzini T, Cardarelli-Leite L, Figueira ERR, Morita F, Domingos FUG, Carnevale FC, de Moura EGH. SpyGlass percutaneous transhepatic cholangioscopyguided lithotripsy of a large intrahepatic stone. Endoscopy. 2017;49(12):E292-E293. https://doi. org/10.1055/s-0043-117943

6. Mendonça EQ, de Oliveira JF, Baba ER, Dias AR, MalufFilho F. Percutaneous transhepatic cholangioscopy for the diagnosis of biliary obstruction in a patient with Roux-en-Y partial gastrectomy. Gastrointest Endosc. 2017;85(1):255256. https://doi.org/10.1016/j.gie.2016.07.057

7. Enochsson L, Swahn F, Arnelo U, Nilsson M, Löhr M, Persson G. Nationwide, population-based data from 11,074 ERCP procedures from the Swedish Registry for Gallstone Surgery and ERCP. Gastrointest Endosc. 2010;72(6):117584, 1184.e1-3. https://doi.org/10.1016/j.gie.2010.07.047

8. Nennstiel S, Weber A, Frick G, Haller B, Meining A, Schmid RM, Neu B. Drainage-related Complications in Percutaneous Transhepatic Biliary Drainage: An Analysis Over 10 Years. J Clin Gastroenterol. 2015;49(9):764-70. https://doi.org/10.1097/MCG.0000000000000275

9. Giovannini M, Moutardier V, Pesenti C, Bories E, Lelong B, Delpero JR. Endoscopic ultrasound-guided bilioduodenal anastomosis: a new technique for biliary drainage. Endoscopy. 2001;33(10):898-900. https://doi. org/10.1055/s-2001-17324

10. Sharaiha RZ, Khan MA, Kamal F, Tyberg A, Tombazzi CR, Ali B, Tombazzi C, Kahaleh M. Efficacy and safety of EUSguided biliary drainage in comparison with percutaneous biliary drainage when ERCP fails: a systematic review and meta-analysis. Gastrointest Endosc. 2017;85(5):904-914. https://doi.org/10.1016/j.gie.2016.12.023

11. Khashab MA, Valeshabad AK, Afghani E, Singh VK, Kumbhari V, Messallam A, Saxena P, El Zein M, Lennon AM, Canto MI, Kalloo AN. A comparative evaluation of EUS-guided biliary drainage and percutaneous drainage in patients with distal malignant biliary obstruction and failed ERCP. Dig Dis Sci. 2015;60(2):557-65. https://doi. org/10.1007/s10620-014-3300-6

12. Khan MA, Akbar A, Baron TH, Khan S, Kocak M, Alastal Y, Hammad T, Lee WM, Sofi A, Artifon EL, Nawras A, Ismail MK. Endoscopic Ultrasound-Guided Biliary Drainage: A Systematic Review and Meta-Analysis. Dig Dis Sci. 2016;61(3):684-703. https://doi.org/10.1007/ s10620-015-3933-0

13. Dhir V, Isayama H, Itoi T, Almadi M, Siripun A, Teoh AYB, Ho KY. Endoscopic ultrasonography-guided biliary and pancreatic duct interventions. Dig Endosc. 2017;29(4):472-485. https://doi.org/10.1111/den.12818

14. Tyberg A, Desai AP, Kumta NA, Brown E, Gaidhane M, Sharaiha RZ, Kahaleh M. EUS-guided biliary drainage after failed ERCP: a novel algorithm individualized based on patient anatomy. Gastrointest Endosc. 2016;84(6):941946. https://doi.org/10.1016/j.gie.2016.05.035

15. Kunda R, Pérez-Miranda M, Will U, Ullrich S, Brenke D, Dollhopf M, Meier M, Larghi A. EUS-guided choledochoduodenostomy for malignant distal biliary obstruction using a lumen-apposing fully covered metal stent after failed ERCP. Surg Endosc. 2016;30(11):5002-5008. https:// doi.org/10.1007/s00464-016-4845-6

16. Minaga K, Kitano M, Yamashita Y, Nakatani Y, Kudo M. Stent migration into the abdominal cavity after EUS-guided hepaticogastrostomy. Gastrointest Endosc. 2017;85(1):263-264. https://doi.org/10.1016/j. gie.2016.03.016

17. Martins FP, Rossini LG, Ferrari AP. Migration of a covered metallic stent following endoscopic ultrasoundguided hepaticogastrostomy: fatal complication. Endoscopy. 2010;42 Suppl 2:E126-7. https://doi. org/10.1055/s-0029-1243911

18. Bailey AA, Bourke MJ, Williams SJ, Walsh PR, Murray MA, Lee EY, Kwan V, Lynch PM. A prospective randomized trial of cannulation technique in ERCP: effects on technical success and post-ERCP pancreatitis. Endoscopy. 2008;40(4):296-301. https://doi. org/10.1055/s-2007-995566 